\title{
Development of a low-cost frequency shift keying signal Transmitter for digital signal processing.
}

\author{
Olumuyiwa Oludare Fagbohun \\ Department of Electrical \& Electronic Engineering, Faculty of Engineering, Ekiti State University, Ado-Ekiti, \\ Ekiti - State, Nigeria.
}

\begin{abstract}
A transmitter consists of an oscillator that generates a carrier frequency followed by a buffer amplifier which provides a high impedance load to minimise drifts, and modulated with an amplified intelligence signal. The development of a low cost frequency shift keying (fsk) transmitter is the concern of this work. The fsk signal forming the base band signal is low - level modulated with a carrier, within the mark and space frequencies. The output from the frequency shift keying circuit at a space frequency of $10 \mathrm{kHz}$ and a mark frequency of $20 \mathrm{kHz}$ was modulated with a $500 \mathrm{kHz}$ single frequency carrier using a diode AM modulating circuit. The transmitter circuit was designed with the use of active devices with few passive components. The measured resonant frequency is $478.63 \mathrm{kHz}$ at $13.427 \mathrm{~dB}$, while the calculated resonant frequency is $485 \mathrm{kHz}$. The calculated carrier power is $1.62 \mathrm{~W}$, and the total power radiated out from the antenna is $2.35 \mathrm{~W}$. The signal noise was calculated to be $5.228 \mathrm{~dB}$ while the measured signal noise was $5.399 \mathrm{~dB}$. The amplified signal is connected to an antenna, with the response of the design simulated on Multism -12 application software. This development can be used to encode digital signals for information transfer in an area within a 5 kilometre radius with precision.
\end{abstract}

Keywords: Frequencies, Fsk signal, Modulator, Op-amp, Transmitter, Transistors.

\section{Introduction}

Transmitters are a major subsystems of a communication network, which accepts the input information, prepares the information for transfer on the transmission link, and raises the output power to a satisfactory level for successful transfer. For a transmitter, a specific frequency (carrier frequency) is generated, which modulate the input base-band signal that contains the information, and increase the power level for coupling to an antenna for transmission $[1,2,3]$. When an electrical information (base-band) signal formed by a frequency shift keying circuit is not compatible with the communications medium, this base-band signal is used to modulate a higherfrequency sine wave signal that is in a frequency (electromagnetic) spectrum that is compatible with the communications medium. This higher-frequency sine wave signal is called a carrier, generated with the use of an RC oscillator circuit. This oscillator outputs the signal to a buffer which isolates itself from the rest of the transmitter circuit, so that the oscillator sees a constant load, and prevents any change in the power stages of the transmitter from being reflected back into the oscillator stage. A circuit that causes a low-frequency base-band signal to modulate a higher-frequency carrier is called a modulator $[3,4]$.

Amplitude modulators (AM) are generally one of two types: low level and high level. In low-level modulation the AM is generated at an early amplifier stage and in high-level modulation the final amplifier stage of a transmitter is modulated. The choice between low-level and high-level modulation is made on the basis of the transmitter power output [5]. In this work, we limit ourselves to the use of FSK, which is a single, low performance form of digital modulation. FSK is a constant - envelope form of angle modulation similar to conventional frequency modulation except that, the modulating signal is binary pulse stream that varies between two discrete voltage levels rather than a continuously changing waveform. With binary FSK, the centre or carrier frequency is shifted (deviated) by the binary input data. Consequently, the output of an FSK modulator is a step function in the frequency domain $[4,6,7]$. As the binary input signal changes from logic to logic and vice versa, the FSK output shifts between two frequencies; a mark or logic 1 frequency and a space or logic 0 frequency. With FSK, there is a change in the output frequency each time the logic condition of the binary input signal changes. Consequently, the output rate of change is equal to the input rate of change. In digital modulation, the rate of change at the right to the modulator is called the bit rate and has the units of bits per second (bps). The rate of change at the output of the modulator is called baud rate or band and is equal to the reciprocal of the time of one output signalling element $[6,8]$. In FSK, the input and output rates of change are equal and thus, the bit rate and baud rate are equal. The block diagram of a low-cost fsk signal transmitter is as shown in Fig 1. 


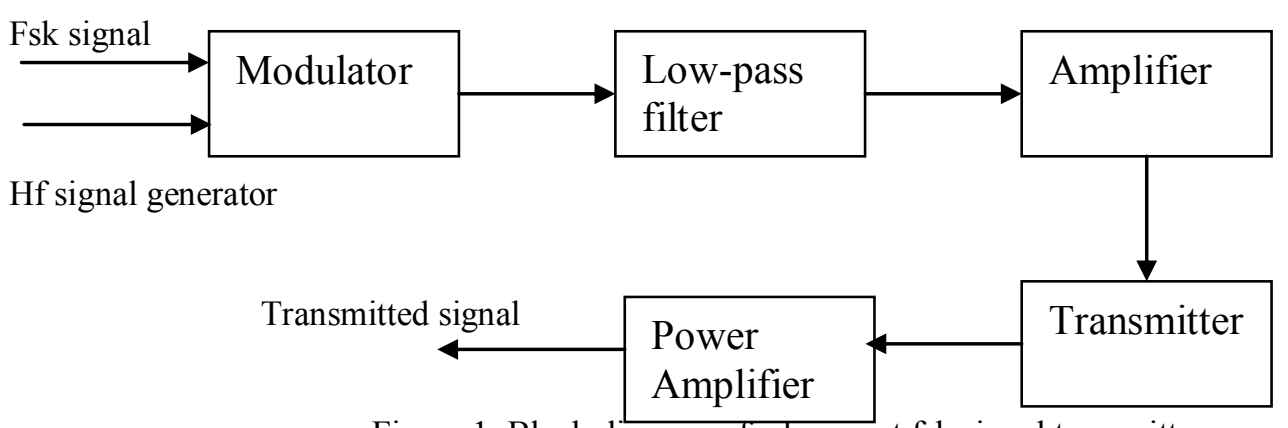

Figure 1: Block diagram of a low-cost fsk signal transmitter.

Low-level modulation does not require much amplification of the modulating signal, and it is more economical for low-power applications than high-level modulation. To amplitude modulate a carrier is to apply the sum of the carrier and the modulating signal to a nonlinear component or circuit that will produce an output that is a function of the square of the input. A diode (or transistor) will accomplish this objective. A diode (or transistor base-emitter junction) has a current (i) that is approximately equal to $I=a V+b V^{2}$; where $\mathrm{V}$ is the voltage across the diode (or transistor base-emitter) and $\mathrm{a}$ and $\mathrm{b}$ are constants $[6,9]$. The resulting output is the carrier frequency and its harmonics, the modulating signal frequencies and their harmonics, an upper carrier sideband, and a lower carrier sideband [10]. In a FSK modulator, $\Delta \mathrm{F}$ is the peak frequency deviation of the carrier and is equal to the difference between the rest frequency and either the mark or space frequency (or half the difference between the $\mathrm{F}_{\mathrm{M}}$ and $\mathrm{F}_{\mathrm{S}}$ ). The peak frequency deviation depends on the magnitude of the modulating signal. In a binary digital signal, all logic 1's or 0's have the same voltage thus, the frequency deviation is constant and always at its maximum value. $F_{a}$ is equal to the fundamental frequency of the binary input which under the worst - case condition (alternating 1's and 0's) is equal to one half of the bit rate. Thus, for FSK modulating index MI ( 1's and 0's) is equal to one half of the bit rate. For FSK

$\mathrm{MI}=\frac{\left(\mathrm{F}_{\mathrm{M}}-\mathrm{F}_{\mathrm{S}}\right) / 2}{\mathrm{~F}_{\mathrm{b}} / 2}=\frac{\mathrm{F}_{\mathrm{M}}-\mathrm{F}_{\mathrm{S}}}{\mathrm{F}_{\mathrm{b}}}$

where; $\left(\mathrm{F}_{\mathrm{M}}-\mathrm{F}_{\mathrm{S}}\right) / 2=$ Peak frequency deviation and $\mathrm{F}_{\mathrm{b}} / 2=$ Fundamental frequency of the binary input signal.

With conventional FM, the bandwidth is directly proportional to the modulation index. Consequently, in FSK, the modulation index is generally kept below 1.0, thus producing a relatively narrowband FM output spectrum [11]. The minimum bandwidth required to propagate a signal is called the minimum Nyquist bandwidth $\left(\mathrm{F}_{\mathrm{n}}\right)$. When modulation is used and double-sided output spectrum is generated, the minimum bandwidth is called the minimum double-sided Nyquist bandwidth or the minimum IF bandwidth $[4,13]$. Because FSK is a form of narrow band frequency modulation, the minimum bandwidth is dependent on the modulation index. For a modulation index between 0.5 and 1, either 2 or 3 sets of significant side frequencies are generated. The minimum bandwidth is two to 3 times the input bit rate $[8,11]$. A parallel band-pass filter tuned to the carrier, with a bandwidth wide enough to pass the carrier and sidebands, will filter out the remaining frequencies. This carrier and two sidebands in the frequency domain will produce an amplitudemodulated carrier in the time domain [12].

\section{Methodology of Research}

2.1. Material Used: The materials used in this work include FET and bi-polar transistors, operational amplifiers in varied configurations and passive components-resistors, capacitors and inductors. A diode 1N4001 was used as a modulating component. A Multism-12 application software was used in the analysis and simulation of the results.

\subsection{Oscillator circuit design}

A crystal oscillator circuit is more appropriate for the design. It has a high degree of frequency stability, and different oscillating frequencies can be obtained by simply replacing one crystal with another, and it remains unaffected by changes in supply and transistor parameters [4,5]. However, in this work, we were unable to get a low valued crystal of about $500 \mathrm{kHz}$ from the software in use- the Multisim -12, thus, we opted for a Wien's bridge Oscillator. A Wien's bridge oscillator is a standard oscillator circuit for generating low frequencies in the range of $20 \mathrm{~Hz}$ to $1 \mathrm{MHz}$ [7]. It has an advantage that it gives an extremely pure sine wave output, good frequency stability, and a highly stabilized amplitude $[6,10]$. The Wien bridge oscillator consists of an RC bridge circuit shown in Figure 2, in which the capacitors $C_{1}$ and $C_{2}$ are assumed to be loss free, and resistances $R_{1}$ and $R_{2}$ are separate resistors. 
The usual relationship for balance gives [8];

$\mathrm{R}_{4}\left\{\mathrm{R}_{1}-\mathrm{j} / \mathrm{wC}_{1}\right\}=\mathrm{R}_{3}\left\{\mathrm{R}_{2} /\left(1+\mathrm{jwC}_{2} \mathrm{R}_{2}\right)\right\}$

If we separate the real and imaginary terms, we have

$\mathrm{R}_{1} \mathrm{R}_{4}+\mathrm{R}_{2} \mathrm{R}_{4} \mathrm{C}_{2} / \mathrm{C}_{1}=\mathrm{R}_{2} \mathrm{R}_{3}$ or $\mathrm{C}_{2} / \mathrm{C}_{1}=\mathrm{R}_{3} / \mathrm{R}_{4}-\mathrm{R}_{1} / \mathrm{R}_{2} \quad \ldots \ldots .2$

and $\mathrm{wR}_{2} \mathrm{R}_{4} \mathrm{C}_{2}-\mathrm{R}_{4} / \mathrm{wC}_{1}=0$ or $\mathrm{w}^{2}=1 / \mathrm{C}_{1} \mathrm{R}_{1} \mathrm{C}_{2} \mathrm{R}_{2}$

with $\mathrm{w}=2 \pi \mathrm{f}$, then $\mathrm{f}=1 / 2 \pi \sqrt{ } \mathrm{C}_{1} \mathrm{R}_{1} \mathrm{C}_{2} \mathrm{R}_{2}$

where $f$ is the frequency of oscillation. If $R_{1}=R_{2}=R$ and $C_{1}=C_{2}=C$, from equation 4 ,

$\mathrm{f}=1 / 2 \pi \mathrm{RC}$

........5

For operation at a frequency of $10 \mathrm{kHz}$, if $\mathrm{R}$ is selected as $10 \mathrm{k} \Omega$, then from equation 4 ,

$\mathrm{C}=\mathrm{C}_{1}=\mathrm{C}_{2}=1 / 2 \pi \mathrm{fR}$

$=1.6 \mathrm{nF}$

For operation at a frequency of $20 \mathrm{kHz}$, if $\mathrm{R}$ is selected as $10 \mathrm{k} \Omega$, then, using equation 6 ,

$\mathrm{C}=0.80 \mathrm{nF}=800 \mathrm{pF}$. The feedback signal in this circuit is connected to the non-inverting (+) input terminal so that the op-amp is working as a non-inverting amplifier. Therefore, the feedback network need not provide any phase shift. The output ac signal of the op-amp amplifier is fed back to point $\mathrm{A}$ of the bridge. The feedback signal, $\mathrm{V}_{\mathrm{f}}$ across the parallel combination $\mathrm{R}_{2} \mathrm{C}_{2}$ is applied to the non-inverting input terminal of the op-amp. The gain of the op-amp amplifier is

$A=1+R_{f} / R_{3}$ where $R_{f}=R_{4}$, and the feedback factor, $\beta$ from Figure 2 is,

$\beta=\mathrm{V}_{\mathrm{f}} / \mathrm{V}_{\mathrm{o}}=\mathrm{Z}_{2} / \mathrm{Z}_{1}+\mathrm{Z}_{2}$

where $Z_{1}=\left(R_{1}+1 / s C_{1}\right)$ and $Z_{2}=R_{2} /\left(1+s R_{2} C_{2}\right)$

putting $Z 1$ and $Z 2$ in equation 7 , and $s=j w$, we get

$\beta=\left(\mathrm{R}_{2} \mathrm{C}_{1} /\left(\mathrm{R}_{2} \mathrm{C}_{2}+\mathrm{R}_{2} \mathrm{C}_{2}+\mathrm{R}_{2} \mathrm{C}_{2}\right)\right.$

for, $\mathrm{R}_{1}=\mathrm{R}_{2}=\mathrm{R}$ and $\mathrm{C}_{1}=\mathrm{C}_{2}=\mathrm{C}$

$\beta=1 / 3$

Since $|\mathrm{A} \beta| \geq 1$ for sustained oscillations, $|\mathrm{A}| \geq 3$; and from equation 7 , $3=1+\mathrm{R}_{4} / \mathrm{R}_{3}$

or $\mathrm{R}_{4}=2 \mathrm{R}_{3}$

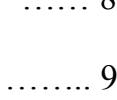

From equation 10 , if $R_{3}$ is selected as $22 k$, then $R_{4}=44 k$.

The resistor $\mathrm{R}_{4}$ was selected as a potentiometer for adjustment, with a value of $50 \mathrm{k}$ and and $\mathrm{R} 3$ as $22 \mathrm{k}$.

If the gain $|\mathrm{A}|>3$, sometimes oscillations keep growing and it may clip the output sine-wave. This problem is eliminated by a practical Wien bridge oscillator with adaptive negative feedback as shown in figure 2. In this circuit, resistor $\mathrm{R}_{4}$ is initially adjusted to give a gain so that oscillations start [9]. The output signal grows in amplitude until the voltage across $\mathrm{R}_{3}$ approaches the cut in voltage of the diode. As the diodes begin to turn on (one for the positive and the other for the negative cycle), the effective feedback resistance $R_{f}$ decreases because the diode is in parallel with the resistance $R_{3}$. This will reduce the gain of the amplifier which in turn lowers the output amplitude. Hence, sustained oscillations can be obtained. Further, if the output falls, the diodes would begin to turn off thereby increasing the $R_{f}$ which in turn increases gain. The values of $R_{1}, R_{2}$ and $C_{1}, C_{2}$ form the frequency adjustment elements for the Wien bridge amplifier circuit, while the $R_{4}, R_{5}$ form the feedback path.

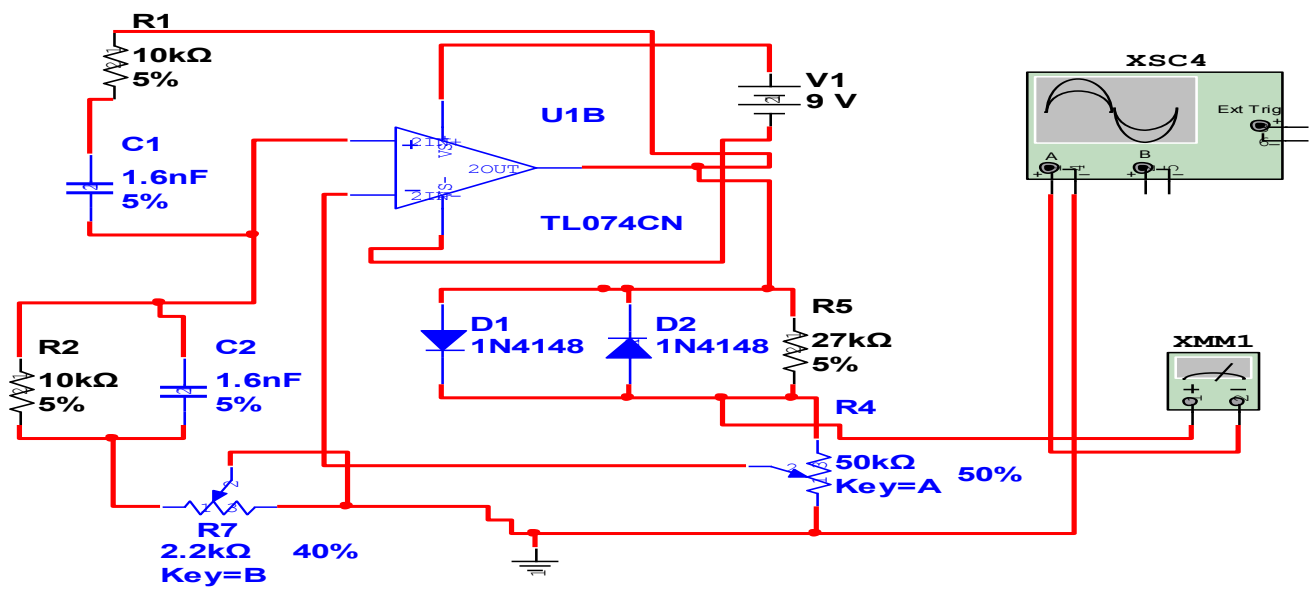

Figure 2: Wien bridge oscillator circuit design 


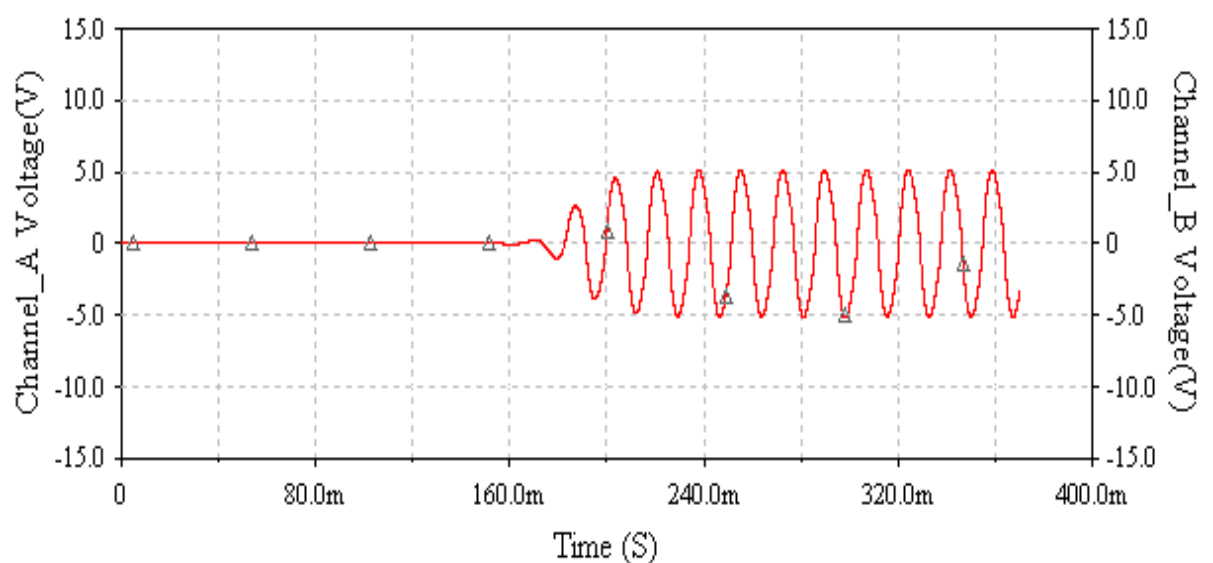

Figure 3: Wien's bridge circuit response at start-up.

\subsection{Design methodology of a low cost fsk signal transmitter}

The coded information forming the baseband frequency is used to switch on two oscillators of predetermined frequencies [12]. The 2 transistors Q1 and Q2 used, are either in a turn-off position (OV) or saturation $(+5 \mathrm{~V})$ with the incoming digital signal of the baseband frequency. The two different frequencies from the collector output of the transistors representing the high - and - low - logic signal frequency of the baseband information forms an input to an op-amp summing circuit U1 via the two input resistors [13]4. The fsk signal developed is fed to a pre op-amp U1 designed to give a voltage gain of 10. Because of the phase inversion of the op-amp adder U1, a voltage follower U2 is connected to the output of the op-amp U1, to give a $360^{\circ}$ phase inversion, to retain the correctness of information. The output from the op-amp U2, is a frequency shift - keying operation signal representing the baseband information required for transfer to the transmitting network. This signal is amplitude modulated with a carrier frequency of $500 \mathrm{kHz}$ using a diode AM modulating circuit. The modulated signal consists of low side frequencies $f_{\mathrm{L} 1}$ and $\mathrm{f}_{\mathrm{L} 2}$, the carrier frequency $[3,13]$, and the upper side frequencies $f_{\mathrm{U} 1}$ and $\mathrm{f}_{\mathrm{U} 2}$ of both the space and mark frequencies of the frequency shift keying signals.

The filter circuit used in this work is basically a band pass filter with the use of parallel resonant circuit, to pass only the low side frequencies of the space and mark, with a high quality factor [8,14], using equations 21 and 22. It is a narrow band filter that passes frequencies in the range of 480-490kHz. To determine the parameters of the parallel resonant circuit, the quality factor $Q$, is $=f_{R} / B W$; where the bandwidth, $B=f_{L 1}$ $-\mathrm{f}_{\mathrm{L} 2}$ (i.e $490-480 \mathrm{kHz}=10 \mathrm{kHz}$ ) and the resonant frequency $\mathrm{f}_{\mathrm{R}}=\sqrt{ } \mathrm{f}_{\mathrm{L} 1} \mathrm{f}_{\mathrm{L} 2}$ is $485 \mathrm{kHz}$. Thus, $\mathrm{Q}$ is $485 / 10$ $=48.5$ which is a narrow band pass filter design specification. For a parallel resonant circuit, $Q=R p / X_{L}$ or $R_{P}$ / $X_{C}$, where $X_{L}=2 \pi f_{R} L$ and $X_{C}=1 / 2 \pi f_{R} C$, and $R p=Q X_{L}$. If $L$ is taken as $10 u H$, then $X_{L}=2 \pi \times 485 \times 103 x$ $10 \times 10-6=30.473$ and therefore $\mathrm{Rp}=48.5 \times 30.473=1488 \Omega$ or $1.5 \mathrm{k} \Omega$. The narrow band pass circuit with the calculated values is as shown in Figure 5. The amplified modulated signal from the modulating circuit is connected to a transmitter. The class B push-pull amplifier is designed for the transmitter is as shown in Fig 6 . Capacitors $\mathrm{C}_{1}, \mathrm{C}_{2}$ and $\mathrm{C}_{3}$ are coupling capacitors to prevent the transistor dc bias voltages from being affected by the input circuit or the load circuit. The amplifier voltage gain is measured by dividing the ac peak to peak output voltage Vo by the ac peak to peak input voltage Vin. Because the push pull in Fig 4, is an emitter follower configuration, the voltage gain should be close to unity.

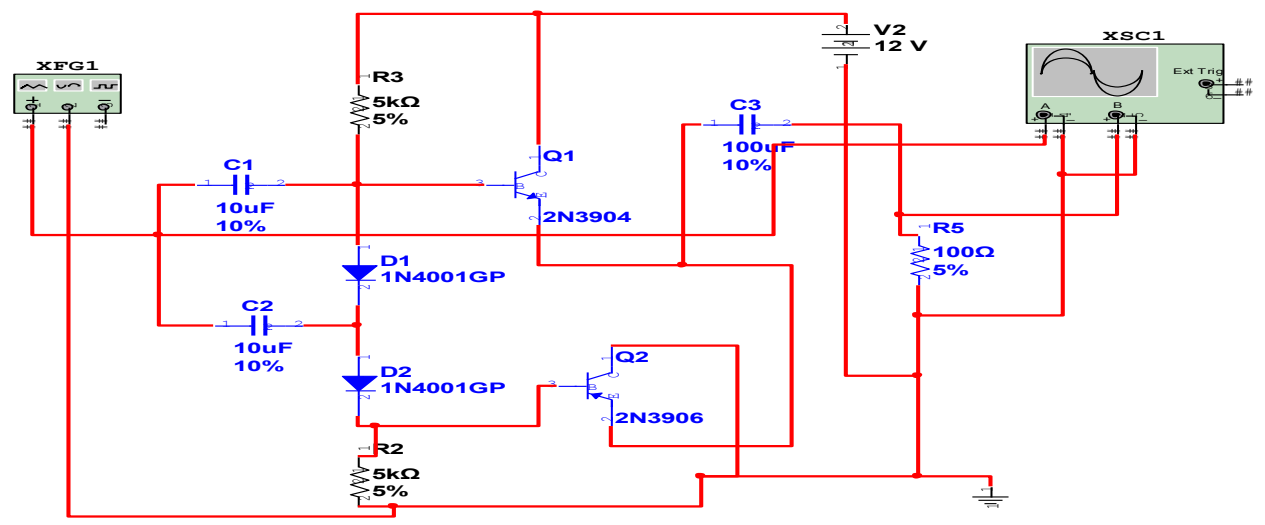

Figure 3: Class B push-pull amplifier design for transmitter 
The amplifier output power $\left(\mathrm{P}_{\mathrm{o}}\right)$ for a class B push- pull amplifier is calculated as follows [14]:

$$
P_{o}=\frac{V^{2}}{R M S_{-}}=\frac{V^{2}}{R_{L}} \frac{O(P-P)}{8 R_{L}}-=\underline{I^{2}} R_{L}
$$

where $\mathrm{V}_{\mathrm{O}(\mathrm{P}-\mathrm{P})}$ is the peak-to-peak output voltage and

$V_{R M S}=\frac{V_{o(p-p)}}{2 \sqrt{2}}$

The percent efficiency $(\eta)$ of an amplifier is equal to the maximum output power $\left(\mathrm{P}_{\mathrm{o}}\right)$ divided by the dc power supplied by the source $\left(\mathrm{P}_{\mathrm{s}}\right)$ times $100 \%$ (Gupta,2003). Therefore,

$$
\begin{aligned}
\eta= & \underline{P}_{o} \times 100 \%=\pi / 4\left(1-\mathrm{V}_{\mathrm{ce}(\min )} / \mathrm{V}_{\mathrm{cc}}\right) \% \\
P_{s} & =78.5(1-1 / 9)=70 \%
\end{aligned}
$$

where $\mathrm{P}_{\mathrm{s}}=\left(\mathrm{V}_{\mathrm{CC}}\right)\left(\mathrm{I}_{\mathrm{CC}}\right)$. The power supply current $\left(\mathrm{I}_{\mathrm{CC}}\right)$ is determined from

$$
\mathrm{I}_{\mathrm{CC}}=\mathrm{I}_{\mathrm{C}(\mathrm{AVG})}=\underline{\underline{\mathrm{I}}_{\mathrm{C}(\mathrm{sat})}}
$$

where $\mathrm{I}_{\mathrm{C}(\text { (sat) }}=\mathrm{V}_{\mathrm{CC}} / 2 \mathrm{R}_{\mathrm{L}}$ and $\mathrm{I}_{\mathrm{C}(\mathrm{AVG})}$ is the average value of the half-wave collector current. Note: $\mathrm{I}_{\mathrm{RB}}$ is normally much less than $\mathrm{I}_{\mathrm{C}(\mathrm{AVG})}$ and can be neglected. The output from the push-pull class $\mathrm{B}$ power amplifier is further amplified before it is connected to a transmitting antenna. To complete the wireless transmission link, each wireless transmitter and receiver requires an antenna. At the transmitter, energy is transferred from the final power amplifier to the antenna, which then radiates energy in the form of electromagnetic waves into the air to produce the wireless transmission.

The total transmitted power is calculated from the equation [10];

Where

$$
\mathrm{P}_{\mathrm{t}}=\mathrm{P}_{\mathrm{c}}\left(1+\mathrm{m}^{2} / 2\right) \ldots
$$

$\mathrm{P}_{\mathrm{c}}=$ Carrier power; $\mathrm{m}=$ modulation index. For a carrier voltage of $9 \mathrm{~V}$, with an impedance of $50 \mathrm{ohms}$,

$\mathrm{P}_{\mathrm{c}}=\mathrm{V}^{2} / \mathrm{R}=81 / 50=1.62 \mathrm{~W}$ and from equation 23 , the transmitted power,

$\mathrm{P}_{\mathrm{t}}=1.62\left(1+(0.95)^{2} / 2\right)=2.35 \mathrm{~W}$

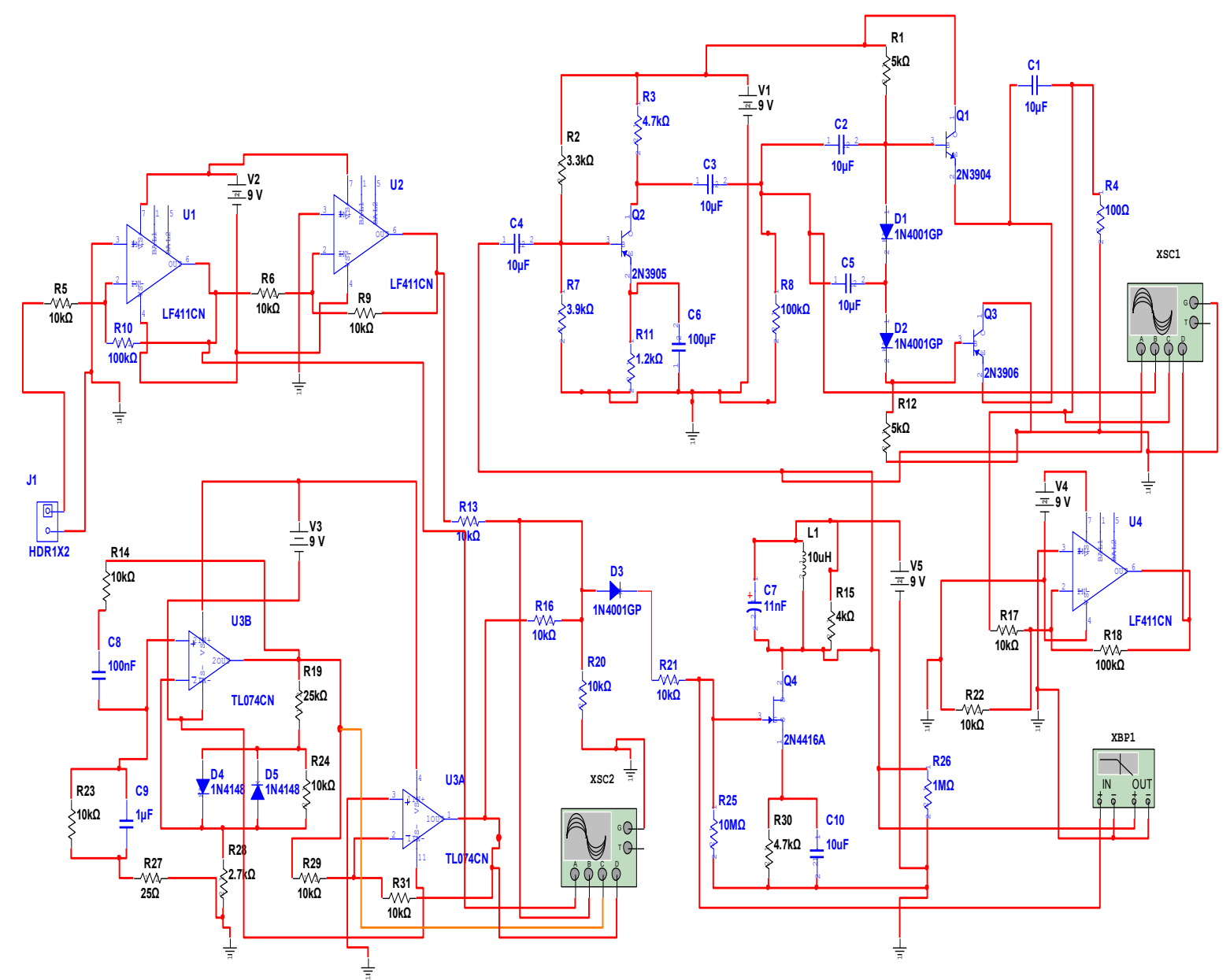

Figure 6. Low cost fsk transmitter circuit design. 


\section{Modulating and transmitter circuit results}

The output from the frequency shift keying circuit at a space frequency of $10 \mathrm{kHz}$ and a mark frequency of $20 \mathrm{kHz}$ [13] connected at junction $\mathrm{J} 1$ was modulated with a $500 \mathrm{kHz}$ single frequency carrier developed with the designed Wien's bridge circuit of Fig 4, using a diode D1 AM modulating circuit of Fig 6 with an output response of Fig 8. A band pass filter network which passes the low side frequencies of the mark and space output response is as shown with the Bode plotter of Fig 7. The measured gain of the filter is as shown in Table 1 .

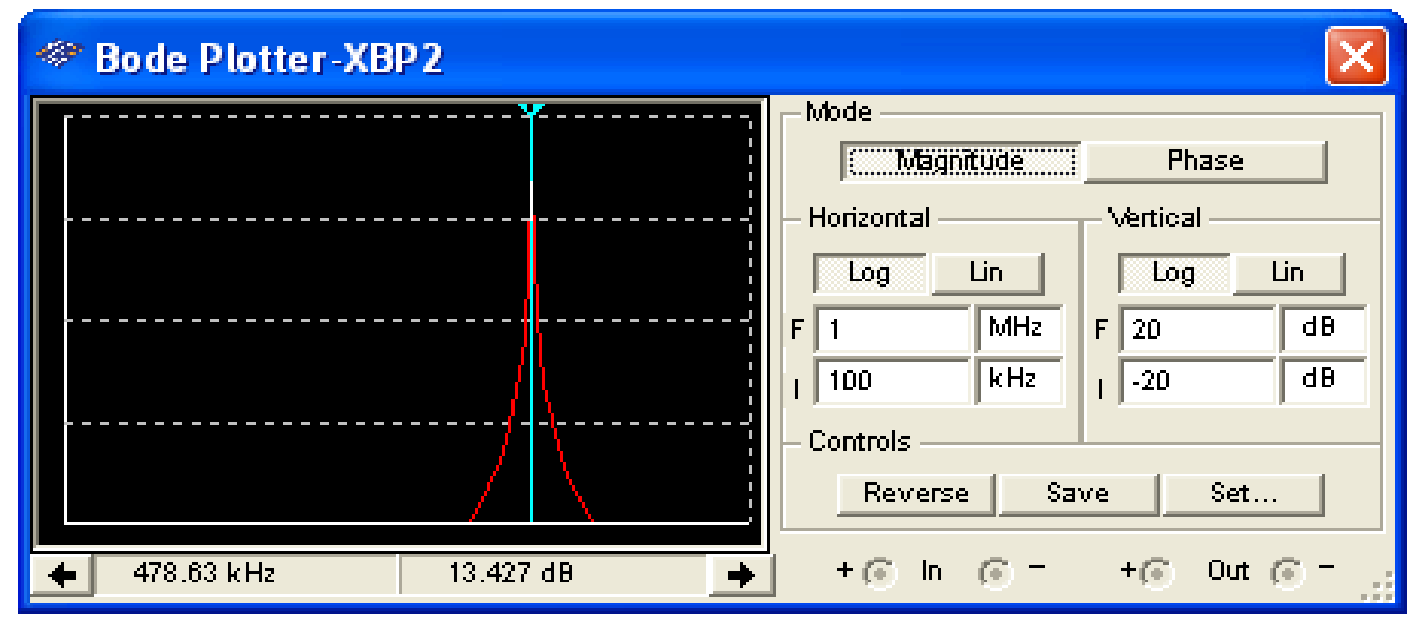

Figure 7: Measured band pass filter circuit response diagram with bode plotter.

Table 1 : Measured Band pass frequencies and gain for resonant frequency of $485 \mathrm{kHz}$.

\begin{tabular}{|l|l|}
\hline Bandpass frequency in $\mathrm{kHz}$ & Measured gain in $\mathrm{dB}$ \\
\hline 459.433 & 0.226 \\
\hline 478.63 & 13.427 \\
\hline 483.553 & 10.977 \\
\hline 488.527 & 7.729 \\
\hline 493.552 & 4.448 \\
\hline 503.758 & -1.210 \\
\hline 508.94 & -2.566 \\
\hline
\end{tabular}

The measured gain of the parallel resonant circuit band pass filter with the use of the circuit design of Fig 6 is as shown in Table 1. The output of the band-pass network formed by the parallel resonant of Fig 6 serves as an input to a common emitter amplifier for a large voltage, current and power gain as shown in Fig 8. The voltage measured at the output of the band-pass network is $20.38 \mathrm{mV}$, with a current of $34 \mathrm{uA}$.. This result is used as an input to a common emitter amplifier. For the common emitter amplifier the calculated base current is $8.3 \mathrm{~mA}$, while the measured current is $10 \mathrm{~mA}$. The calculated voltage gain and power gain is $\mathrm{Av}=+156$ and $\mathrm{Gp}$ $=51.93 \mathrm{~dB}$, while the measured voltage gain as seen in Fig 9 is +167 and power gain $\mathrm{Gp}=52.23 \mathrm{~dB}$. The signal noise was calculated to be 5.228 while the measured signal noise as was $5.399 \mathrm{~dB}$. The measured resonant frequency is $478.63 \mathrm{kHz}$ at $13.427 \mathrm{~dB}$ as shown in Fig 7, while the calculated resonant frequency is $485 \mathrm{kHz}$. From Table 1, it can be seen that the gain for the calculated resonant frequency lies between 10.977 and 7.729. The difference is due to the approximations in the value of the capacitors and inductors used in the circuit. Fig 8a shows the measured FSK signal output, being modulated by a diode modulator to a carrier frequency of 500 $\mathrm{kHz}$ of Fig 8b, with an output as shown in Fig 8c. The signal consists of frequencies in the range of 480-520 $\mathrm{kHz}$. The measured carrier voltage is $2.643 \mathrm{~V}$, with the output voltage of the modulator at $4.497 \mathrm{~V}$. The diode modulator output forms the input to the tuned circuit FET amplifier whose output voltage measured at its collector is $4.214 \mathrm{~V}$. This is shown with the measured values in Fig 9a. The parallel resonant circuit band pass network of the tuned FET amplifier as shown in Fig 6 pass frequencies in the range of $475-495 \mathrm{kHz}$, while rejecting any other. The output from the tuned circuit is connected to the push-pull emitter circuit through a common emitter amplifier. Because the push-pull configuration of the transmitter circuit is an emitter follower, the voltage gain is close to unity. The output of the transmitter circuit is further amplified by the op-amp amplifier before being connected to the transmitting antenna. The $\%$ modulation for the FSK signal sent with the carrier at $500 \mathrm{kHz}$ is calculated as $95 \%$ (i.e $4.95 \mathrm{~V}$ for both the signal and carrier amplitude ). The calculated carrier power $\left(\mathrm{P}_{\mathrm{c}}=\mathrm{V}^{2} / \mathrm{R}\right)$ is $1.62 \mathrm{~W}$, and the total power radiated out from the antenna is $2.35 \mathrm{~W}$ using equation 23 . This is the energy transferred to the antenna of $50 \Omega$ load impedance for radiation and its percent efficiency is $70 \%$. 


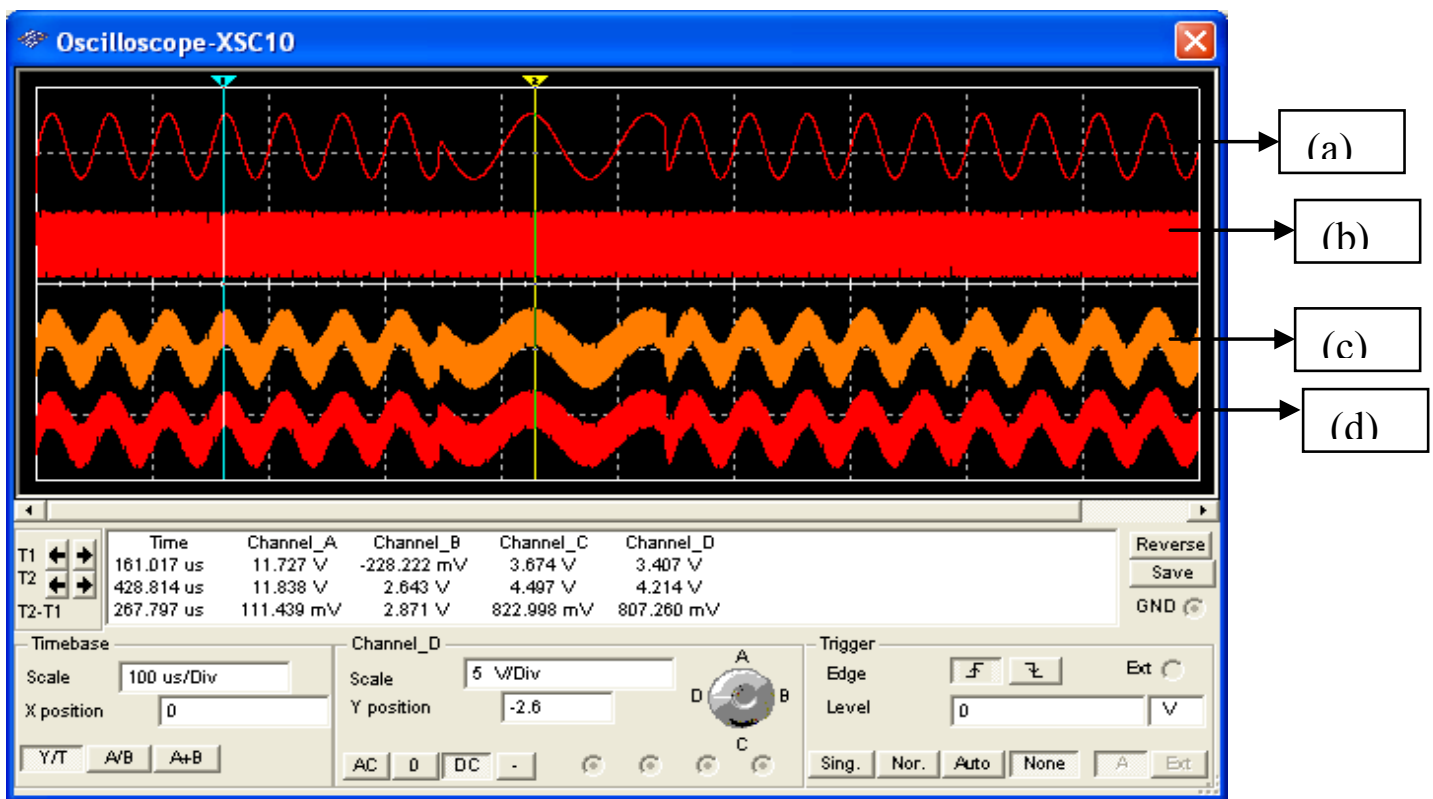

Figure 8:a).FSK signal output b). The carrier signal c). The modulator output, and d). The band pass filter network output.

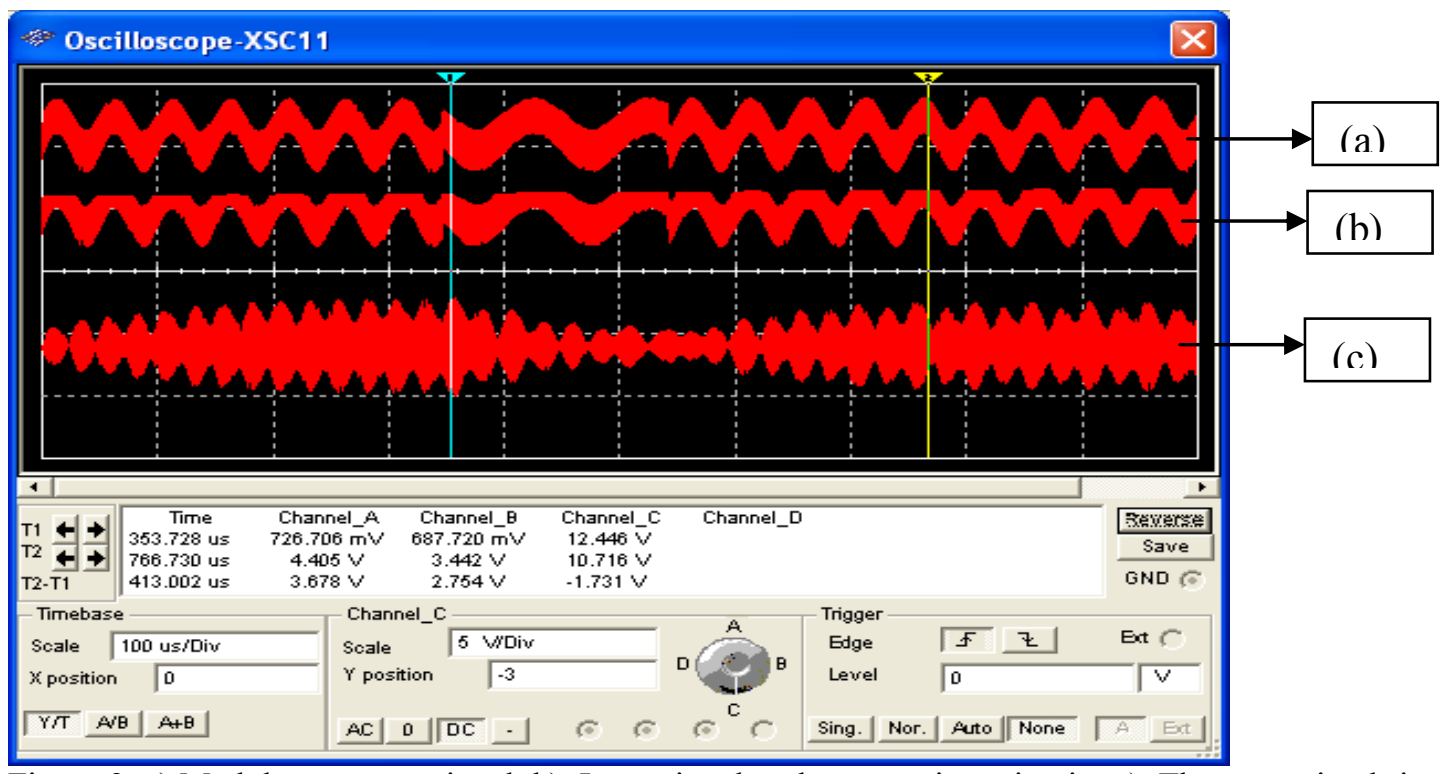

Figure 9: a) Modulator output signal; b). Input signal to the transmitter circuit ; c). The transmitted signal.

\section{Conclusion}

The band pass filter with a parallel resonant used passed only the low side frequency of the space and mark, with a quality factor of 48.5 which is in line with the narrow band design specification. The efficiency of the design amplifier is $70 \%$ and the total transmitted power is $2.35 \mathrm{~W}$ which is transferred from the power amplifier to the antenna and radiates energy in the form of electromagnetic waves into the air for wireless transmission. This fsk signal transmitter can be used to encode digital signals for information transfer in an area within a 5 kilometre radius with precision in an area of small jurisdiction, and being recommended for electronic policing and security information relay system with a low cost of manufacture. 


\section{References}

[1]. W.J. Hudson, and J. Luecke, Basic Communications Electronics Radio-Shark R 62-1336, Master Publishing Inc, Lincolnwood, Illinois; 1999

[2]. J.S. Beasley, and G.M. Miller, Modern Electronic Communication, $8^{\text {th }}$ edition, Pearson Education inc; 2005.

[3]. W. Tomasi, Advanced Electronic Communication System; Prentice Hall inc, New Jersey, $2^{\text {nd }}$ Edition, 1992

[4]. E.S. Batho, Electronic design principles, $1^{\text {st }}$ edition, Prentice Hall inc, New Jersey, 1992.

[5]. A.K. Theraja, and B.L. Theraja, A textbook of electrical Technology, $23^{\text {rd }}$ ed., S.Chand and Company, 2003

[6]. H.R. Berube, Learning Electronics communications through experimentation using electronic workbench multisim;Prentice Hall inc. 2002

[7]. H. Baggot, Practical filter design, Elektor electronics journal, April, 1989, 22-29

[8]. B.R. Gupta, Electronics and Instrumentation, $3^{\text {rd }}$ edition, S.Chand and Company,2003.

[9]. M.L. Anand, Electronic principles - Devices and Circuits, $6^{\text {th }}$ edition, S.Chand and Company, 1999.

[10]. H.G. Brierley, Telecommunications Engineering, Third edition, John Wiley \& Sons Inc, 2002.

[11]. R.S. Sheda, A textbook of Digital Electronics, $1^{\text {st }}$ edition, S.Chand and company ltd;.2004.

[12]. R.F.W. Coates, Modern Communication Systems , $2^{\text {nd }}$ edition, Prentice Hall, New Jersey; 1982

[13]. M. Schwartz, Information Transmission, modulation, and Noise. $3^{\text {rd }}$ edition, McGraw-Hill Series in Electrical Engineering, 1980.

[14]. J. Milman, and C.C. Halkias, Integrated Electronics, : Analog and Digital circuits and Systems, 30 ${ }^{\text {th }}$ edition, Mc-Graw-Hill book company;2004.

[15]. R.J. Brad, and E.S. Carlos, Frequency shift keying (FSK) modulator using MEM's switch,2004 www.goggle .fsk . Visited on 12 February 2006

[16]. Subir Kumar Sarka : Operational Amplifiers and their applications, Revised $1^{\text {st }}$ edition, S.Chand and Company.2003.

[17]. R.J. Tocci : Digital systems : Principles and applications ; 8th Edition, Prentice hall int. inc., London, 2002.

[18]. O.O. Fagbohun, Development of a low cost frequency shift keying (fsk) modulator with transistor switching ; International Journal of Engineering , 4(2), 2010, 249-255

[19]. O.O. Fagbohun, Development of a receiver circuit for medium frequency shift keying signals, IOSR Journal of Electrical and Electronic Engineering (IOSR-JEEE) e-ISSN: 2278-1676, p-ISSN2320-3331, 9(2), 2014, 28-35

[20]. Wikipedia, (2006c) : fsk circuits . The free encyclopaedia, http://en.wikipedia.org / wiki/ fsk circuits

[21]. Wikipedia, (2006e) : Low power transmitters. The free encyclopaedia, http://en.wikipedia.org / wiki/ transmitters/ low_power 